\title{
Autorreporte de salud de los adultos mayores en México, 2012-2018
}

\author{
Daniel Lozano Keymolen* \\ Sergio Cuauhtémoc Gaxiola Robles Linares ${ }^{\star *}$ \\ Alejandro Martínez Espinosa ${ }^{\star \star *}$
}

El objetivo de esta investigación fue analizar los efectos de determinados factores sociodemográficos y de la salud en las trayectorias del autorreporte de salud de las personas mayores en México. Con datos del Estudio Nacional de Salud y Envejecimiento en México 20122018, se estimaron las trayectorias mediante modelos de estados latentes de Markov. Los resultados indican que, tanto en mujeres como en hombres hay tres trayectorias, las cuales fueron constantes incluso ajustando por variables sociodemográficas y de la salud. Sin embargo, el arreglo residencial, la multimorbilidad o los síntomas depresivos tuvieron efectos negativos sobre la probabilidad de un autorreporte de salud positivo. Estos resultados sugieren que, si bien debe profundizarse el análisis de los factores asociados con las trayectorias del autorreporte de salud, los arreglos residenciales, las múltiples enfermedades crónicas o la depresión tienen efectos longitudinales en la salud autoevaluada de los adultos mayores en México.

Palabras clave: Autorreporte de salud. Estados latentes. Envejecimiento. Enfermedades crónicas. México.

\footnotetext{
* Centro de Investigación y Estudios Avanzados de la Población de la Universidad Autónoma del Estado de México, Toluca, México (daniel.lozkey@gmail.com; https://orcid.org/0000-0003-1086-7233).

** Centro de Investigación y Estudios Avanzados de la Población de la Universidad Autónoma del Estado de México, Toluca, México (serobles99@gmail.com; https://orcid.org/0000-0002-7782-9058).

${ }^{* \star \star}$ Centro de Investigación y Estudios Avanzados de la Población de la Universidad Autónoma del Estado de México, Toluca, México (alejandro.mtz.esp@gmail.com; https://orcid.org/0000-0002-6937-1200).
} 


\section{Introducción}

El autorreporte de salud (ARS) es uno de los indicadores subjetivos más utilizados para determinar el estado y las dinámicas de salud a nivel individual y poblacional, ya que permite inferir parte del razonamiento y de la biología de las personas al evaluar su salud y los eventos relacionados con esta (IDLER; BENYAMINI, 1997; MIILUNPALO et al., 1997). El ARS se determina de forma global al preguntar En la actualidad, ¿podría decir que su salud es...?, con opciones de respuesta: excelente, muy buena, buena, regular o mala, o de forma relativa En comparación con individuos de su edad y sexo, ¿podría decir que su salud es...?, con respuestas que van desde mejor, más o menos igual hasta peor. Sin embargo, en ambos casos existen adecuaciones a las respuestas (IDLER; BENYAMINI, 1997; JYLHÄ, 2011).

El ARS es un predictor de condiciones o padecimientos graves, de la funcionalidad (JOHNSON; WOLINSKY, 1993; WOLINSKY; TIERNEY, 1998; YOSHIMITSU et al., 2017) y de la mortalidad aun después de controlar por variables de la salud y sociodemográficas (FALCONER; QUESNEL-VALLEÉ, 2016; KAPLAN; CAMACHO, 1983). El ARS permite incluso determinar el uso de servicios de salud también en personas sin enfermedades (JYLHÄ, 2011; MIILUNPALO et al., 1997) y es una de las mediciones de salud más empleadas tanto en encuestas nacionales como en ensayos clínicos por su facilidad de aplicación y asociación con medidas de salud objetivas (IDLER; BENYAMINI, 1997; JYLHÄ, 2011).

Sin embargo, la asociación del ARS como predictor de la morbilidad o de la mortalidad podría relacionarse con los métodos, las variables o las percepciones y las creencias de la población de estudio, pues se han estimado diferencias entre grupos étnicos o culturas que se relacionarían con la disponibilidad de información y de servicios de atención a la salud, así como con factores lingüísticos y de interpretación de las categorías de respuesta del ARS (LEE; SCHWARZ; GOLDSTEIN, 2014; MOLINA, 2017). También, es posible que el ARS dependa de la información y de las condiciones específicas y expectativas de salud que los individuos valoran (JYLHÄ, 2011; ZAJACOVA; HUZURBAZAR; TODD, 2017).

En todo caso, sobre los procesos de generación del ARS se han propuesto diversas hipótesis que se relacionan con múltiples variables, así como con los contextos sociales y los procesos que los subyacen. Entonces, la hipótesis de la esponja sostiene que la percepción de la salud captura todos los indicadores y experiencias de la salud anteriores; la hipótesis de la intervención plantea que la autoevaluación de la salud influye en los comportamientos de salud que a su vez afectan los resultados de salud; la hipótesis de los recursos argumenta que el ARS refleja la disponibilidad de apoyos internos o externos que afectan a los resultados de salud; la hipótesis de la trayectoria plantea que los individuos consideran cambios anteriores en su salud para determinar su percepción actual, por lo cual sería una forma de anticipación de cambios o del declive de la salud, o incluso de la mortalidad (IDLER; BENJAMINI, 1997; WOLINSKY; TIERNEY, 1998). 
En el contexto de los procesos de envejecimiento poblacional, la hipótesis de la trayectoria permite identificar factores socioeconómicos y de la salud que inciden en las necesidades de salud de las personas mayores, así como de los elementos que interactúan entre las personas y los medios en los cuales habitan, incluidos distintos elementos subjetivos (FALCONER; QUESNEL-VALLEÉ, 2016; WOLINSKY; TIERNEY, 1998). Además, el análisis longitudinal del ARS se ha asociado como un indicador de la mortalidad, de cambios que anteceden al diagnóstico de enfermedad, de las contingencias en salud o del soporte 0 capital social disponible que, en el caso de las personas mayores, supone una importante fuente de conocimiento sobre los elementos que intervienen en las trayectorias del ARS (MILLER; WOLINSKY, 2007; PINILLOS-FRANCO; KAWACHI, 2017).

En el caso de los adultos mayores, se ha propuesto que estos basan sus evaluaciones de la salud en comparaciones sociales, antecedentes de salud y mortalidad de individuos de edades similares (CHEN et al., 2016; JYLHÄ, 2011). Si bien algunos estudios apuntan a que en las personas mayores la forma relativa suele ser más precisa (CAMELO, GIATTI; BARRETO, 2014; SUH: DOCTOR, 2013), otros trabajos entienden que la forma global sería un mejor indicador (SARGENT-COX; ANSTEY-LUSZCZ, 2010; VUORISALMI; LINTONEN; JYLHÄ, 2006). En general, la evidencia no es concluyente y, en el caso de las personas mayores, parte de la literatura considera equivalentes a las formas global y relativa del ARS (CHEN et al., 2016).

El ARS es uno de los indicadores subjetivos más utilizados para determinar el estado, las dinámicas y los factores que inciden en la salud de las personas mayores. Esto es importante en países que, como México, experimentan acelerados procesos de envejecimiento, pues, para $2010,9,42 \%$ de la población tenía sesenta y más años de edad y se proyecta que sea $22,5 \%$ en 2050 (CONAPO, 2018). El envejecimiento de la estructura etaria en México se relaciona con una elevada morbilidad por enfermedades como diabetes (24,3\% en 2012) o condiciones como limitaciones en las actividades básicas de la vida diaria (ABVD) (26,9\% en 2012) (MANRIQUE-ESPINOZA et al., 2013). Aspectos como los anteriores se asocian con mayores necesidades de cuidado para las personas mayores en un contexto de aumento del número de adultos mayores que residen solos (6,48\% en 2015) (INEGI, 2015).

Sobre el ARS, entre las personas mayores en México, se ha estimado que los desocupados o aquellos con enfermedades crónicas tienden a evaluar su salud en forma negativa (GALLEGOS, et al., 2006). Otros trabajos reportan peores evaluaciones de la salud de las mujeres o que la valoración positiva disminuye con la edad (PALLONI et al., 2003; AGUILAR-PALACIO et al., 2018).

Analizar de forma longitudinal el ARS o su trayectoria permitiría determinar las variables que anteceden a los cambios en la valoración de la salud y que se relacionan con modificaciones en la morbilidad, en parte de los comportamientos en la salud o el apoyo y recursos sociales disponibles de los adultos mayores en México. Entonces, el objetivo de esta investigación fue analizar los efectos de determinados factores sociodemográficos y de la salud en las trayectorias del autorreporte de salud de los adultos mayores en México. 


\section{Método}

\section{Muestra}

Los datos se obtuvieron del Estudio Nacional de Salud y Envejecimiento en México (ENASEM). El ENASEM es una encuesta longitudinal con entrevista basal en $2001 \mathrm{y}$ reentrevistas en 2003, 2012, 2015 y 2018. Se trata además de una encuesta con diseño probabilístico y representatividad a niveles nacional y urbano-rural, que en la ronda 2001 entrevistó a más de quince mil personas en poco más de once mil hogares. El ENASEM es un estudio que ha actualizado la muestra original, pues en la ronda de 2012 se agregaron 5.896 nuevos entrevistados de las cohortes de nacimientos 1952-1962 y para la ronda del 2018 se adicionaron personas de las cohortes de nacimiento 1963-1968 (WONG; MICHAELS-OBREGON, 2019).

Para esta investigación solo se emplearon las rondas 2012-2018 del ENASEM ya que el espacio temporal en cada una de estas tres rondas es de tres años. La muestra de análisis se compuso de entrevistas directas de personas con sesenta y más años de edad en el ENASEM 2012, pues no se utilizaron entrevistados sustitutos dado que estos no son informativos del ARS del entrevistado principal (LI; HARRIS; LU, 2015). Solo se seleccionaron los casos con datos completos para las variables del estudio en las tres rondas de análisis: ENASEM 2012 (t1), ENASEM 2015 (t2) o ENASEM 2018 (t3). Entonces, de las 10.170 entrevistas directas de personas mayores o con sesenta y más años de edad en 2012 no se tomó el $11,3 \%$ de observaciones, por ausencia de valores en las variables de análisis en t1, t2 o t3. Hecho lo anterior, se analizaron 9.019 casos de la siguiente forma: 5.911 observaciones de sobrevivientes en $\mathrm{t} 2$ y $\mathrm{t} 3$ a los cuales se aplicó el análisis de trayectorias del ARS (55,8\% mujeres y 44,2\% hombres). Luego, se describen las distribuciones del ARS en 1.434 defunciones tanto en la ronda 2012, como en la entrevista anterior a la captación de la muerte ( $47,9 \%$ mujeres y $52,1 \%$ hombres). Por último, se caracterizan 1.674 observaciones con pérdida de seguimiento ( $56,5 \%$ mujeres y $43,6 \%$ hombres).

\section{Variables de análisis}

Variable dependiente

Se definió como ARS global y se construyó según la pregunta global y que en las tres rondas analizadas del ENASEM es igual: Ahora tengo algunas preguntas sobre su salud, ¿Diría usted que su salud es...? con opciones de respuesta: 1) excelente, 2) muy buena, 3) buena, 4) regular, 5) mala. Aunque el ARS se evalúa de forma dicotómica o incluso de manera categórica (JYLHÄ, 2011), en este análisis se analiza con una variable ordinal de tres categorías ( 1 = ARS bueno (excelente/muy buena/buena), 2 = ARS regular (regular), 3 = ARS malo (mala)). Esta categorización se concretó con un análisis previo en el que las respuestas excelente o muy buena mostraron distribuciones porcentuales $<5 \%$ en $\mathrm{t} 1, \mathrm{t} 2$ y t3, por lo que se agregaron a la categoría ARS bueno. Además, se estimaron los estados latentes y estos no se modifican respecto de usar tres o cinco categorías del ARS. 


\section{Variables de control}

El Cuadro 1 expone las variables sociodemográficas y de la salud relacionadas con el ARS entre las personas mayores que se utilizan como controles estadísticos. Se agregan las categorías analíticas de acuerdo con la revisión bibliográfica hecha.

\section{CUADRO 1}

Variables de control, referencias teóricas y categorías analíticas para evaluar el autorreporte de salud en adultos mayores. ENASEM 2012, 2015, 2018

\begin{tabular}{|c|c|c|}
\hline Variable & Referencias teóricas & $\begin{array}{l}\text { Categorías } \\
\text { analíticas }\end{array}$ \\
\hline Situación laboral & $\begin{array}{l}\text { Existe evidencia de los efectos del estatus laboral en el ARS, pues la } \\
\text { desocupación se relaciona con determinados estresores psicosociales } \\
\text { que afectan la salud en forma negativa (AGUILAR-PALACIO et al., 2018; } \\
\text { OVERLAND et al., 2006). }\end{array}$ & $\begin{array}{l}\text { Variable dicotómica: } \\
\text { 1) trabaja, 2) no } \\
\text { trabaja. }\end{array}$ \\
\hline $\begin{array}{l}\text { Situación } \\
\text { conyugal }\end{array}$ & $\begin{array}{l}\text { Las personas casadas o unidas tienen mejores evaluaciones del } \\
\text { estado de salud que las viudas o divorciadas. Al respecto, se ha } \\
\text { planteado que las diferencias en la percepción de la salud se } \\
\text { asocian con elementos como el apoyo y el control social o el estrés, } \\
\text { que incentivan conductas negativas entre quienes no están unidos } \\
\text { (BERDAHL; MCQUILLAN, 2018; JOUNG et al., 1995). }\end{array}$ & $\begin{array}{l}\text { Variable categórica: } \\
\text { 1) casado/unido, } \\
\text { 2) viudo/ } \\
\text { divorciado/ } \\
\text { separado, } \\
\text { 3) soltero. }\end{array}$ \\
\hline $\begin{array}{l}\text { Arreglo } \\
\text { residencial }\end{array}$ & $\begin{array}{l}\text { El aumento del número de personas adultas mayores que viven en } \\
\text { hogares con un número reducido de integrantes se ha asociado con } \\
\text { peores evaluaciones de la salud, ya que el número de integrantes del } \\
\text { hogar se relaciona con la disponibilidad de recursos o apoyo y soporte } \\
\text { social (MEADOWS, 2009; YOSHIMITSU et al., 2017). }\end{array}$ & $\begin{array}{l}\text { Variable categórica: } \\
\text { 1) familia nuclear, } \\
\text { 2) familia ampliada, } \\
\text { 3) vive solo. }\end{array}$ \\
\hline Multimorbilidad & $\begin{array}{l}\text { Con autorreportes de diagnóstico de diabetes, hipertensión arterial, } \\
\text { enfermedades respiratorias, cáncer, embolia cerebral, artritis o } \\
\text { enfermedades del corazón se evaluó la multimorbilidad, variable } \\
\text { empleada para el estudio del ARS, pues la presencia de EC se asocia } \\
\text { con ARS malos o muy malos (ARIOKIASAMY; UTTAMAHCARYA, 2015; } \\
\text { MARTELL-CLAROS et al., 2011). }\end{array}$ & $\begin{array}{l}\text { Variable categórica: } \\
\text { 1) sin EC, 2) al } \\
\text { menos una EC, } \\
\text { 3) dos o más EC/ } \\
\text { multimorbilidad. }\end{array}$ \\
\hline $\begin{array}{l}\text { Dependencia } \\
\text { funcional }\end{array}$ & $\begin{array}{l}\text { Las restricciones en la movilidad física se relacionan con ARS malos } \\
\text { (JOHNSON; WOLINSKY, 1993; YOSHIMITSU et al., 2017), por lo cual se } \\
\text { evaluó la necesidad de asistencia en las ABVD según el índice de Katz } \\
\text { (KATZ et al., 1963). }\end{array}$ & $\begin{array}{l}\text { Variable categórica: } \\
\text { 1) sin restricciones } \\
\text { en } A B V D, 2) \text { una } \\
\text { restricción en } \\
\text { ABVD, 3) dos o más } \\
\text { restricciones en } \\
\text { ABVD. }\end{array}$ \\
\hline $\begin{array}{l}\text { Síntomas } \\
\text { depresivos }\end{array}$ & $\begin{array}{l}\text { Una parte considerable de la literatura relaciona la presencia de } \\
\text { síntomas depresivos o depresión con malos o muy malos ARS } \\
\text { (AMBRESIN et al., 2014; WEXLER et al., 2012). En el ENASEM, } \\
\text { los síntomas depresivos se estimaron a partir de la versión del } \\
\text { Cuestionario de } 9 \text { reactivos del CES-D, que ha sido validado y muestra } \\
\text { una alta consistencia interna ( } \alpha \text { de Cronbach = 0.86) para definir un } \\
\text { punto de corte } \geq 5 \text { como positivo para síntomas depresivos (AGUILAR- } \\
\text { NAVARRO et al., 2007). }\end{array}$ & $\begin{array}{l}\text { Variable dicotómica: } \\
\text { 1) sin síntomas } \\
\text { depresivos: } \\
\text { < } 5 \text { positivos, } \\
\text { 2) síntomas } \\
\text { depresivos: } \geq 5 \\
\text { puntos positivos. }\end{array}$ \\
\hline $\begin{array}{l}\text { Consumo de } \\
\text { tabaco }\end{array}$ & $\begin{array}{l}\text { Dado que las personas con tabaquismo suelen reportar peores } \\
\text { evaluaciones del estado de salud que quienes no consumen tabaco, } \\
\text { se evaluaron los efectos del tabaco en el ARS (CAMELO; GIATTI; } \\
\text { BARRETO, 2014). }\end{array}$ & $\begin{array}{l}\text { Variable categórica } \\
\text { 1) no fuma, 2) antes } \\
\text { fumaba, 3) fuma. }\end{array}$ \\
\hline $\begin{array}{l}\text { Edad } \\
\text { Edad2 }\end{array}$ & $\begin{array}{l}\text { Diversas investigaciones indican que, según incrementa la edad, la } \\
\text { valoración positiva de la salud disminuye como consecuencia de } \\
\text { los efectos de los factores de riesgo en la salud y de determinados } \\
\text { estresores socioeconómicos (JOHNSON; WOLINSKY, 1993; ROHLFSEN; } \\
\text { JACOBS KRONENFELD, 2014). }\end{array}$ & $\begin{array}{l}\text { Variable continua } \\
\text { que incluye un } \\
\text { término cuadrado. }\end{array}$ \\
\hline
\end{tabular}

Fuente: Elaboración propia. 


\section{Análisis estadístico}

Análisis descriptivo

Se graficaron las distribuciones del ARS global acorde con la situación de la observación en $\mathrm{t} 1, \mathrm{t} 2$ o t3: sobrevivientes, defunciones entre rondas o pérdidas de seguimiento (Figura 1). Además, se estimaron las distribuciones para la variable ARS global y las covariables en $\mathrm{t} 1, \mathrm{t} 2$ y $\mathrm{t} 3$, y se probaron diferencias estadísticas entre las distribuciones para mujeres y hombres según pruebas $x 2$ para las variables categóricas y un análisis de varianza (ANOVA) para la edad (Tabla 1).

\section{Trayectorias del ARS}

Para determinar las trayectorias en el ARS se han aplicado modelos de regresión ajustando por variables individuales, familiares o sociales (BERDAHL; MCQUILLAN, 2018; MILLER; WOLINSKY, 2007), así como modelos de trayectorias ajustando por covariables (ROHLFSEN; JACOBS KRONENFELD, 2014). En esta investigación, las trayectorias del ARS se estimaron según un modelo latente de Markov (MLM), dado que es una técnica que, basada en los procesos de Markov, permite definir los estados latentes que determinan las trayectorias en el ARS de los individuos, así como los efectos de las covariables y en las probabilidades de transición temporales.

En un MLM como el estimado (BARTOLUCCI; FACOMENI; PENNONI, 2012): sea el ARS que se expresa en $\mathbf{j}=3$ respuestas ordinales medidas $T=3$ veces (ENASEM, 2012, 2015, 2018), entonces $Y_{j}^{(t)}$ es cada una de las respuestas individuales con $c_{j}$ categorías. El MLM supone la existencia de un estado latente $U=\left(U^{(1)}, \ldots, U^{(T)}\right)$ que tiene efectos sobre el ARS según un proceso de Markov de primer orden de tiempo homogéneo expresado en un conjunto de estados latente $\{1,2, ., k\}$. Bajo el supuesto de independencia, los vectores respuesta del ARS $Y^{(1)}, \ldots, Y^{(T)}$ son independientes del proceso latente $U$. Es decir que todas las respuestas individuales $Y_{j}^{(t)}$ son independientes de $U^{(t)}$. Los parámetros del modelo de medición de los estados latentes son las probabilidades condicionales del ARS $\phi_{\mathrm{y} \mid \mathrm{u}}=f_{Y^{(t)} \mid U^{(t)}}(\mathrm{y} \mid \mathrm{u})$ con $t=1, \ldots, T, u=1, \ldots, k, y=0, ., c-1$; las probabilidades iniciales según los estados latentes se definen como $\pi_{\mathrm{u}}=f_{U^{(1)}}(\mathrm{u})$, con $u=1, ., k$, y las probabilidades de transición entre los estados latentes son $\pi_{u \mid \bar{u}}^{(t)}=f_{U^{(t)} \mid U^{(t-1)}}(u \mid \bar{u}) \operatorname{con} t=2, \ldots \hat{u}, T, u=1, ., k \operatorname{con} t=2, ., T, y u=1, ., k$.

Entonces, con un primer modelo de medición se determinó el número de estados latentes presentes en la matriz $Y_{j}^{(t)}$ para la variable ARS global. Los estados latentes definen las trayectorias del ARS de acuerdo con $c_{j}$, que, en este caso, son homogéneos en el tiempo y balanceados en el número de observaciones en $\mathrm{t} 1, \mathrm{t} 2$ y $\mathrm{t} 3$. Con el modelo se calcularon tres conjuntos de estimadores: los valores $\hat{\phi}_{y \mid u}$ dicen cómo cada estado latente depende del verdadero nivel del ARS considerando el error de medición; los estimadores $\hat{\pi}_{u}$ determinan las proporciones de la muestra que inician en cada estado latente, y los valores $\hat{\pi}_{u \mid \bar{u}}$ informan sobre los cambios de los individuos entre los niveles del ARS. En un segundo modelo se estimó la heterogeneidad no observada ajustando el primer modelo 
por las covariables sociodemográficas y de la salud, y se seleccionó el modelo con el menor valor del BIC (Tabla 2).

Hecho lo anterior, los efectos de las covariables se parametrizaron con modelos logit ordinal proporcionales por lo que en una primera regresión (modelo 1 ajustado por covariables proporcionales), se calculó la proporcionalidad de las variables mediante el estadístico score-test longitudinal $(p \geq 0,05)$ y solo se incluyeron las covariables que cumplen con el supuesto de proporcionalidad. Luego, se construyó otra regresión (modelo 2 ajustado por covariables totales), que incluye todas las covariables seleccionadas y determina los efectos de estas en las trayectorias definidas (Tabla 3).

Entonces, un modelo logit se expresa como $\log \frac{\phi_{y \mid u x}^{(t)}+\cdots+\phi_{c-1 \mid u x}^{(t)}}{\phi_{y \mid u x}^{(t)}+\cdots+\phi_{y-1 \mid u x}^{(t)}}=\mu_{y}+\alpha_{u}+X^{T} \beta$, donde el vector $X^{(t)}$ contiene los valores de las covariables en T (Cuadro 1), $\mu_{y}$ define los puntos de corte y $\alpha_{u}$ son los puntos de soporte de los estados latentes. Los efectos de las covariables $(\beta)$ se estimaron por máxima-verosimilitud $l(\theta)=\sum_{i=1}^{n} \log f_{\tilde{Y} \mid \tilde{X}}\left(\tilde{y}_{i} \mid \tilde{X}_{i}\right)$ a partir del algoritmo expectación-maximización en el cual $\tilde{X}_{i}$ e $\tilde{y}_{i}$ proporcionan los vectores del ARS y de las covariables. Todos los modelos se construyeron con procesos iterativos de selección de estados latentes considerando que $\phi_{C-1 \mid 1}<\ldots<\phi_{C-1 \mid k}$, se evaluó su ajuste a los datos con el criterio de Schwarz (BIC) y se seleccionó el modelo con el menor valor del BIC. Se empleó la razón de momios (RM) como estadístico de interpretación y, para determinar la posible presencia de multicolinealidad entre las covariables, en t1 se estimó el factor de inflación de la varianza (FIV <10).

\section{Análisis de defunciones}

El análisis comparó las distribuciones del ARS global en t1 entre los sobrevivientes y los fallecidos, así como el ARS en t1 y la última ronda de captación de quienes murieron. No se incluyeron las muertes en el MLM, dado que este solo se emplea para observaciones balanceadas en T (BARTOLUCCI et al., 2012).

Análisis de pérdida de seguimiento

Al igual que entre quienes murieron, se caracterizan las distribuciones del ARS global en las observaciones que tuvieron pérdida de seguimiento con las distribuciones en 2012 de quienes sobrevivieron a las rondas analizadas del ENASEM.

En tanto que tanto el ARS como las variables de control seleccionadas para el análisis han mostrado diferencias entre mujeres y hombres, los análisis se estratificaron por sexo (PALLONI et al., 2003; AGUILAR-PALACIO et al., 2017). Los datos se procesaron con el software $\mathrm{R}$ y el uso del paquete LMest (BARTOLUCCI; PANDOLFI, 2017). 


\section{Resultados}

La Figura 1 muestra seis gráficos por sexo. En los gráficos de los sobrevivientes, entre mujeres no existen cambios en el ARS bueno. Sin embargo, hay un aumento de la categoría ARS regular entre rondas. Para los hombres, el ARS bueno disminuye, aunque de manera similar que las mujeres los hombres reportaron un aumento gradual de $A R S$ regular. En ninguno de los dos sexos se encontraron cambios en la categoría ARS malo. Si se comparan las distribuciones del ARS entre mujeres y hombres, en 2012 el ARS bueno expone diferencias significativas entre rondas $(p<0,050)$, y una situación similar se estimó para la categoría del ARS malo ( $\mathrm{p}<0,05)$. Sin embargo, para el ARS regular no se hallaron diferencias significativas, excepto en 2012 ( $p<0,050)$.

Al analizar los ARS de las defunciones, se encuentra que para mujeres que fallecieron entre 2012 y 2015 , el ARS global se distribuyó en $22 \%$ bueno, 48,2\% regulary $29,8 \%$ malo, lo cual contrasta con los sobrevivientes, entre los que en t1 el 29,5\% declaró ARS bueno, $55,6 \%$, ARS regulary $14,9 \%$, una salud mala. Al estimar diferencias se obtuvo significancia estadística (< 0,050), lo cual permite concluir que un mayor porcentaje de mujeres que fallecieron entre las rondas 2012 y 2015 del ENASEM reportó un ARS malo que aquellas que sobrevivieron. Para t2, entre mujeres que fallecieron $21,9 \%$ tuvo un ARS bueno, 46,4\% regulary $31,7 \%$, malo. En este sentido, se observa que un mayor porcentaje de mujeres que luego murieron había declarado un ARS malo que el de sus contrapartes sobrevivientes.

Entre los hombres que fallecieron entre las rondas 2012 y 2015 del ENASEM, 26\% habían declarado un ARS bueno, 49,7 regulary 24,4\%, malo. Al contrastar estos porcentajes con los de los hombres en 2012 y que sobrevivieron a las tres rondas, una mayor proporción de fallecidos había reportado un ARS malo ( $\mathrm{p}$ 0,050). Luego, cuando se comparó la distribución del ARS entre quienes sobrevivieron a las rondas del ENASEM y de quienes murieron entre 2015 y 2018 , fue notorio que estos habían reportado un mayor porcentaje de salud mala (ARS malo sobrevivientes $=9,8 \%$ y ARS malo en muertes $=28,2 \%, p<0,050$ ).

El análisis de pérdida de seguimiento de quienes fueron entrevistados en el ENASEM 2012 indicó que el 29,5\% de las mujeres sobrevivientes declaró un ARS bueno en 2012 y aquellas cuyo seguimiento se perdió para la ronda 2015 significaron un 32,7\% ( $<<0,050$ ), mientras que los casos de pérdida de seguimiento entre 2015 y 2018 tuvieron un $26,6 \%$ de ARS bueno en 2012. Para los hombres sobrevivientes, 40,3\% declaró en 2012 un ARS bueno, mientras quienes no tuvieron seguimiento en 2015 lo hicieron en un 42,7\% ( $<<0,050)$, y de los hombres que no fueron reentrevistados en la ronda de 2018, un 38,2\% declaró una salud buena en 2012 ( $p<0,050$ ) (Figura 1). 
FIGURA 1

Distribuciones del ARS global en mujeres y hombres entrevistados en el ENASEM de 2012 y sobrevivientes a las rondas 2015 y 2018, defunciones entre rondas y pérdidas de seguimiento entre rondas

Sobrevivientes entre rondas
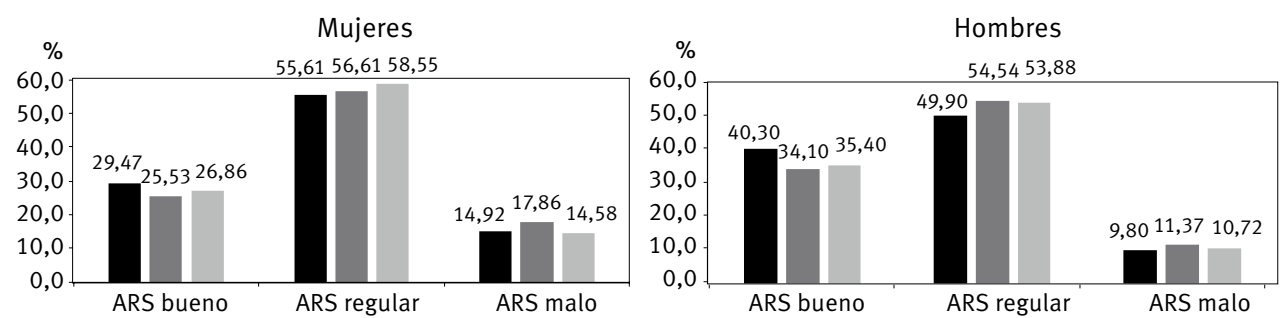

Sobrevivientes 2012 Sobrevivientes 2015 Sobrevivientes 2018

Defunciones entre rondas

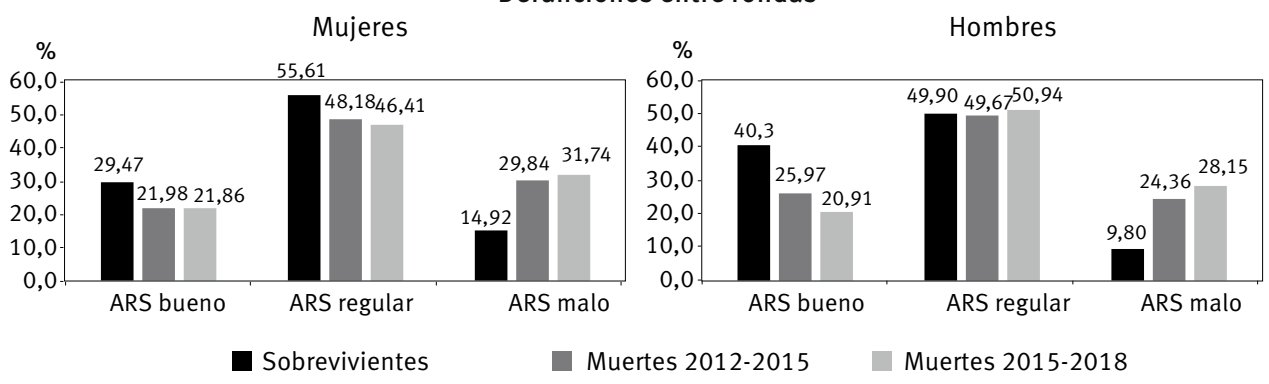

Pérdida de seguimento
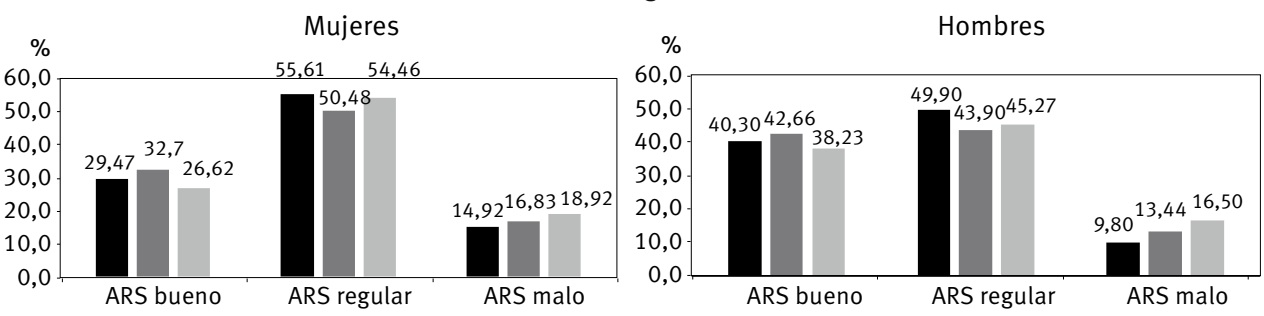

Sobrevivientes

Sin seguimiento 2012-2015

Sin seguimiento 2015-2018

Fuente: Elaboración propia a partir de las 5.911 observaciones de entrevistados en el ENASEM 2012 y sobrevivientes a las rondas 2015 y 2018; de las 1.434 defunciones captadas y los 1.674 casos sin seguimiento entre rondas (ENASEM, 2020).

Nota: Las distribuciones originales de la variable del ARS global en mujeres: $\mathrm{t} 1$ : excelente $=1,2 \%$, muy buena= $3,0 \%$, buena= $25,2 \%$, regular $=55,6 \%$, mala $=14,9 \%$; t 2 : excelente $=1,9 \%$, muy buena $=1,9 \%$, buena $=21,6 \%$, regular $=56,7 \%$, mala $=18,0 \%$; 3 : excelente $=1,6 \%$, muy buena $=2,9 \%$, buena $=22,4 \%$, regular $=58,6 \%$, mala $=14,6 \%$.

Las distribuciones originales de la variable del ARS global en hombres: t1: excelente $=3,1 \%$, muy buena $=5,0 \%$, buena $=32,1 \%$, regular $=49,9 \%$, mala $=9,8 \%$; t2: excelente $=2,8 \%$, muy buena $=3,7 \%$, buena $=27,2 \%$, regular $=54,8 \%$, mala $=11,5 \%$; $\mathrm{t} 3$ : excelente $=2,9 \%$, muy buena $=4,5 \%$, buena $=28,0 \%$, regular $=53,9 \%$, mala $=10,7 \%$.

En la Tabla 1 se muestran las distribuciones de las covariables en $\mathrm{t} 1, \mathrm{t} 2$ y $\mathrm{t} 3$ por sexo. Los resultados exponen amplias diferencias, pues según la situación laboral se observan diferencias características del mercado laboral en México, ya que las mujeres declararon en menor proporción estar trabajando en 2102, 2015 o 2018 ( $p<0,050$ ), aunado a que se encontró una disminución en la participación de las personas sobrevivientes a las rondas 
del ENASEM. La disminución de la participación en la fuerza laboral de las personas mayores se asocia con aspectos como la jubilación, el aumento del autoempleo o la inserción en negocios propios o familiares que en gran parte no se declaran como actividad laboral (CEPAL/OIT, 2018).

Según la situación conyugal, las mujeres reportaron estar casadas o unidas, así como también divorciadas o viudas en mayores proporciones que los hombres en las tres rondas analizadas $(p<0,050)$. En cuanto a los arreglos residenciales, mayores porcentajes de hombres que de mujeres reportaron residir en familias extendidas ( $p<0,050)$, si bien porcentajes más altos de mujeres residían solas ( $p<0,050)$. Estos resultados se han expuesto con otros datos, ya que, en 2015, un 6,5\% de las personas mayores en México vivía en un hogar unipersonal (INEGI, 2015).

TABLA 1

Distribuciones de las variables de control para los modelos de estados latentes de Markov. ENASEM 2012, 2015 y 2018

\begin{tabular}{|c|c|c|c|c|c|c|c|c|c|}
\hline \multirow[t]{2}{*}{ Variable } & \multicolumn{2}{|c|}{$\mathrm{t} 1$} & \multirow[t]{2}{*}{ Sig. } & \multicolumn{2}{|c|}{$\mathrm{t} 2$} & \multirow[t]{2}{*}{ Sig. } & \multicolumn{2}{|c|}{ t3 } & \multirow[t]{2}{*}{ Sig. } \\
\hline & Mujeres & Hombres & & Mujeres & Hombres & & Mujeres & Hombres & \\
\hline Situación laboral & & & $<0,001$ & & & $<0,001$ & & & $<0,001$ \\
\hline Trabaja & 16,1 & 49,8 & & 16,7 & 49,1 & & 11,9 & 37,4 & \\
\hline No trabaja & 83,9 & 50,2 & & 83,3 & 50,9 & & 88,1 & 62,6 & \\
\hline Situación conyugal & & & $<0,001$ & & & $<0,001$ & & & $<0,001$ \\
\hline Casado/unido(a) & 55,4 & 83,9 & & 50,1 & 79,9 & & 44,8 & 76,7 & \\
\hline Divorciado/viudo(a) & 39,1 & 13,7 & & 45,1 & 18,3 & & 49,6 & 20,7 & \\
\hline Soltero(a) & 5,5 & 2,3 & & 4,8 & 1,8 & & 5,6 & 2,6 & \\
\hline Arreglo residencial & & & $<0,001$ & & & $<0,001$ & & & $<0,001$ \\
\hline Familia extendida & 54,9 & 64,6 & & 52,5 & 62,4 & & 55,6 & 65,8 & \\
\hline Familia nuclear & 38,4 & 30,7 & & 39,7 & 33,8 & & 34,9 & 28,9 & \\
\hline Vive solo(a) & 6,7 & 4,7 & & 7,8 & 3,8 & & 9,6 & 5,4 & \\
\hline Multimorbilidad & & & $<0,001$ & & & $<0,001$ & & & $<0,001$ \\
\hline $\operatorname{Sin} \mathrm{EC}$ & 28,1 & 44,2 & & 23,1 & 38,8 & & 22,0 & 37,6 & \\
\hline Una EC & 38,1 & 35,1 & & 36,7 & 34,6 & & 34,9 & 31,1 & \\
\hline $2+E C$ & 33,8 & 20,7 & & 40,2 & 26,6 & & 43,1 & 31,3 & \\
\hline Dependencia funcional & & & $<0,001$ & & & $<0,001$ & & & $<0,001$ \\
\hline $\operatorname{Sin} A B V D$ & 84,2 & 91,2 & & 80,8 & 88,4 & & 82,3 & 88,8 & \\
\hline Una ABVD & 9,8 & 5,6 & & 10,4 & 7,2 & & 5,6 & 4,3 & \\
\hline $2+A B V D$ & 6,0 & 3,2 & & 8,8 & 4,4 & & 12,1 & 6,9 & \\
\hline Síntomas depresivos & & & $<0,001$ & & & $<0,001$ & & & $<0,001$ \\
\hline Sí & 40,1 & 21,2 & & 38,7 & 22,4 & & 39,0 & 23,4 & \\
\hline No & 59,9 & 78,8 & & 61,3 & 77,6 & & 61,0 & 76,6 & \\
\hline Consumo de tabaco & & & $<0,001$ & & & $<0,001$ & & & $<0,001$ \\
\hline Fuma & 5,1 & 17,5 & & 4,6 & 16,1 & & 3,8 & 13,9 & \\
\hline Antes fumaba & 12,9 & 43,4 & & 16,1 & 48,9 & & 16,0 & 47,9 & \\
\hline Nunca ha fumado & 82,0 & 38,9 & & 79,3 & 35,0 & & 80,2 & 38,2 & \\
\hline Edad (media) & 68,1 & 68,5 & 0,004 & 71,2 & 71,7 & 0,004 & 74,2 & 74,7 & 0,005 \\
\hline
\end{tabular}

Fuente: Elaboración propia a partir de las 5.911 observaciones de entrevistados en el ENASEM 2012 y sobrevivientes a las rondas 2015 y 2018 . Mujeres $n=3.298$, Hombres $n=2.613$ (ENASEM, 2020).

Nota: $E C=$ enfermedad crónica; $A B V D$ = actividades básicas de la vida diaria; Sig. = significancia del estadístico de prueba. 
Para las covariables de la salud, las mujeres entrevistadas en el ENASEM 2012 y sobrevivientes a las rondas 2015 y 2018 declararon tener dos o más EC en mayor proporción que los hombres ( $\mathrm{p}<0,050$ ). Tal situación puede relacionarse no solo con las elevadas prevalencias de EC como diabetes (24,3\%) e hipertensión (40\%) entre los adultos mayores en México (MANRIQUE-ESPINOZA et al., 2013), sino también con que las mujeres suelen acudir en mayor medida a los servicios de salud preventivos y de diagnóstico de EC (SALINAS, 2015).

Respecto de las limitaciones en las ABVD, las mujeres declaran tener más restricciones que los hombres ( $p<0,050)$. Además, los porcentajes de mujeres con síntomas depresivos fueron mayores que en los hombres según el instrumento validado para el ENASEM ( $p<0,050)$. De hecho, en 2012, un porcentaje más alto de mujeres tenía síntomas depresivos significativos, lo cual es similar a lo estimado en otros lugares (PICAZZO-PALENCIA, 2016). Por último, los hombres fuman tabaco en mayor porcentaje que las mujeres ( $p<0,050)$, si bien existe una reducción en la prevalencia de consumo de tabaco entre 2012 y 2018 para ambos sexos, situación asociada con el descenso del tabaquismo entre las cohortes más viejas del país (FRANCO-MARINA, 2007).

En la Tabla 2 se exponen los resultados del modelo de medición para determinar y caracterizar los estados latentes (k) en la variable ARS global, así como los parámetros $\hat{\phi}_{y \mid u}, \hat{\pi}_{u}$ y $\pi_{u \mid \hat{u}}^{(t)}$. Entonces, se determinaron tres estados latentes $(\mathrm{k}=3)$ en mujeres (BIC = 17.591,61), y se definió el estado latente 1 como de disminución de la salud o de ARS bueno a ARS regular ( $\hat{\phi}_{y \mid u}=0,710$ y 0,256$)$, el estado latente 2 como de regular salud $\left(\hat{\phi}_{y \mid u}=0,796\right)$, mientras que el estado latente 3 expresaría un decaimiento general de la salud o de $A R S$ regular a malo $\left(\hat{\phi}_{y \mid u}=0,230\right.$ y 0,757$)$. El estado latente de regular salud concentró la mayor proporción de personas según las probabilidades iniciales $\left(\hat{\pi}_{u}=0,565\right)$. Por otro lado, según las probabilidades de transición se observó una alta permanencia en los estados definidos $\left(\pi_{u \mid \hat{u}}^{(t)}=0,844,0,955\right.$ y 0,885$)$, incluso cuando se agregaron los efectos de las covariables, tanto en el modelo 1 ajustado por covariables proporcionales (BIC = $17.102,28$ y $\pi_{u \mid \hat{u}}^{(t)}=0,913,0,943$ y 0,801$)$, como en el modelo 2 ajustado por covariables totales $\left(\mathrm{BIC}=57.552,19\right.$ y $\pi_{u \mid \hat{u}}^{(t)}=0,814,0,865$ y 0,960$)$.

La Tabla 2 resume también los resultados para los hombres entre los que se determinaron tres estados latentes $(\mathrm{k}=3)(\mathrm{BIC}=13.749,46)$. Se definió el estado latente 1 como de mejoramiento de la salud $\left(\hat{\phi}_{y \mid u}=0,396\right.$ y 0,552$)$, mientras que el estado latente 2 $\left(\hat{\phi}_{y \mid u}=0,758\right.$ y 0,174$)$ podría definirse como de regular salud. El estado latente 3 se clasificó como de decaimiento de la salud al pasar de un ARS regular a malo $\left(\hat{\phi}_{y \mid u}=0,174\right.$ y 0,825). En este caso, el estado latente 2 o de salud regular tuvo la mayor proporción de hombres en el ENASEM de $2012\left(\hat{\pi}_{u}=0,527\right)$. Las probabilidades de transición indicaron que, para los estados latentes definidos, los valores $\left.\hat{\phi}_{y \mid u}=0,882,0,956,0,868\right)$ indican una alta permanencia en el estado definido, pues al incorporar las covariables se mantuvieron los tres estados definidos tanto en el modelo 1 ajustado por covariables proporcionales 
$\left(\mathrm{BIC}=13.586,52\right.$ y $\pi_{u \mid \hat{u}}^{(t)}=0,943,0,750$ y 0,928$)$ como en el modelo 2 ajustado por covariables totales $\left(\mathrm{BIC}=51.462,47\right.$ y $\pi_{u \mid \hat{u}}^{(t)}=0,956,0,831$ y 0,899$)$.

TABLA 2

Matrices de probabilidades de transición para los estados latentes sin ajustar y ajustados según la variable ARS global. ENASEM, 2012, 2015 y 2018

\begin{tabular}{|c|c|c|c|c|c|c|c|c|c|}
\hline Variables & \multicolumn{3}{|c|}{ Modelo sin ajustar } & \multicolumn{3}{|c|}{$\begin{array}{c}\text { Modelo } 1 \text { ajustado por } \\
\text { covariables proporcionales }\end{array}$} & \multicolumn{3}{|c|}{$\begin{array}{l}\text { Modelo } 2 \text { ajustado por } \\
\text { covariables totales }\end{array}$} \\
\hline $\begin{array}{l}\text { Selección de } \\
\text { estados }\end{array}$ & $\mathrm{BIC}$ & & & $\mathrm{BIC}$ & & & $\mathrm{BIC}$ & & \\
\hline \multicolumn{10}{|l|}{ Mujeres } \\
\hline$k=1$ & $19.142,70$ & & & $18.182,45$ & & & $87.685,99$ & & \\
\hline$k=2$ & $17.963,89$ & & & $17.306,80$ & & & $77.343,10$ & & \\
\hline$k=3$ & $17.591,61$ & & & $17.102,28$ & & & $57.552,19$ & & \\
\hline \multicolumn{10}{|l|}{$\hat{\pi}_{u}$} \\
\hline$k=1$ & 0,151 & & & 0,295 & & & 0,218 & & \\
\hline$k=2$ & 0,565 & & & 0,582 & & & 0,200 & & \\
\hline$k=3$ & 0,284 & & & 0,123 & & & 0,582 & & \\
\hline$\hat{\phi}_{y \mid u}$ & $k=1$ & $k=2$ & $k=3$ & $k=1$ & $k=2$ & $k=3$ & $k=1$ & $k=2$ & $k=3$ \\
\hline $\begin{array}{l}\text { Excelente/ } \\
\text { muy buena/ } \\
\text { buena }\end{array}$ & 0,710 & 0,085 & 0,013 & & & & & & \\
\hline Regular & 0,256 & 0,796 & 0,230 & & & & & & \\
\hline Mala & 0,033 & 0,119 & 0,757 & & & & & & \\
\hline $\begin{array}{l}\text { Matriz de } \\
\text { transición }\end{array}$ & $k=1$ & $k=2$ & $k=3$ & & & & & & \\
\hline$k=1$ & 0,844 & 0,147 & 0,009 & 0,913 & 0,063 & 0,024 & 0,814 & 0,110 & 0,077 \\
\hline$k=2$ & 0,032 & 0,955 & 0,013 & 0,027 & 0,943 & 0,030 & 0,102 & 0,865 & 0,033 \\
\hline$k=3$ & 0,000 & 0,115 & 0,885 & 0,079 & 0,120 & 0,801 & 0,035 & 0,005 & 0,960 \\
\hline \multicolumn{10}{|l|}{ Hombres } \\
\hline & $\mathrm{BIC}$ & & & & & & & & \\
\hline$k=1$ & $14.806,41$ & & & $14.453,44$ & & & $77.373,52$ & & \\
\hline$k=2$ & $13.869,20$ & & & $13.669,43$ & & & $67.488,61$ & & \\
\hline$k=3$ & $13.749,46$ & & & $13.586,52$ & & & $51.462,47$ & & \\
\hline \multicolumn{10}{|l|}{$\hat{\pi}_{u}$} \\
\hline$k=1$ & 0,118 & & & 0,621 & & & 0,559 & & \\
\hline$k=2$ & 0,527 & & & 0,068 & & & 0,075 & & \\
\hline$k=3$ & 0,355 & & & 0,311 & & & 0,366 & & \\
\hline$\hat{\phi}_{y \mid u}$ & $k=1$ & $k=2$ & $k=3$ & & & & & & \\
\hline $\begin{array}{l}\text { Excelente/ } \\
\text { muy buena/ } \\
\text { buena }\end{array}$ & 0,552 & 0,067 & 0,001 & & & & & & \\
\hline Regular & 0,396 & 0,758 & 0,174 & & & & & & \\
\hline Mala & 0,053 & 0,176 & 0,825 & & & & & & \\
\hline $\begin{array}{l}\text { Matriz de } \\
\text { transición }\end{array}$ & $k=1$ & $k=2$ & $k=3$ & & & & & & \\
\hline$k=1$ & 0,882 & 0,083 & 0,035 & 0,943 & 0,027 & 0,030 & 0,956 & 0,001 & 0,044 \\
\hline$k=2$ & 0,037 & 0,956 & 0,007 & 0,195 & 0,750 & 0,055 & 0,003 & 0,831 & 0,166 \\
\hline$k=3$ & 0,000 & 0,132 & 0,868 & 0,072 & 0,001 & 0,928 & 0,067 & 0,034 & 0,899 \\
\hline
\end{tabular}

Fuente: Elaboración propia a partir de las 5.911 observaciones de entrevistados en el ENASEM 2012 y sobrevivientes a las rondas 2015 y 2018 (ENASEM, 2020).

Nota: $\mathrm{BIC}=$ Criterio de Información Bayesiana. 
Definidas las tres trayectorias del ARS global para mujeres y hombres, se estimaron los efectos de las covariables y determinar la posible presencia de heterogeneidad no observada que influiría en las trayectorias definidas. Como se comentó en la metodología, se ajustaron dos modelos de regresión: la primera regresión se ajustó por las covariables que mostraron evidencia estadística de no violar el supuesto de proporcionalidad, mientras que en la segunda regresión se ajustó por todas las covariables analizadas. Además, las pruebas del FIV indicaron que la multicolinealidad no es un factor que afecte las estimaciones.

En la Tabla 3 se resumen los efectos de las covariables en los estados latentes ARS global. En el caso de las mujeres, solo arreglo residencial, situación conyugal, multimorbilidad y consumo de tabaco fueron proporcionales $\left(X^{2}=6,93, p=0,140\right)$. Para los hombres, las covariables con proporcionalidad fueron arreglo residencial, situación conyugal, dependencia funcional y consumo de tabaco $\left(X^{2}=8,93, p=0,063\right)$. Estas variables se emplean en el modelo 1 ajustado por covariables proporcionales tanto en mujeres como en hombres.

Cuando se analizan los resultados del modelo 1 ajustado por covariables proporcionales, en las mujeres, estar solteras incrementa su probabilidad de declarar una salud buena $(\beta=0,544, R M=1,72)$. También, pertenecer a una familia extendida es un factor que aumenta la probabilidad de un ARS bueno $(\beta=0,304, \mathrm{RM}=1,36)$. Estos resultados se mantienen para el modelo 2 ajustado por covariables totales, ya que las mujeres que habitan en familias extendidas tienen mayor probabilidad de declarar un ARS bueno $(\beta=0,302, R M=1,35)$, e incluso estar solteras aumenta su propensión a un ARS positivo $(\beta=0,550, R M=1,73)$.

Relacionado con las variables de la salud y para el modelo 1 ajustado por covariables proporcionales, entre las mujeres tener una $\mathrm{EC}(\beta=-1,077, \mathrm{RM}=0,34)$ o multimorbilidad $(\beta=-2,089, \mathrm{RM}=0,12)$ disminuye la probabilidad de tener un ARS positivo. Estos resultados son consistentes en el modelo 2 ajustado por covariables totales, pues, tener una EC $(\beta=-0,955, \mathrm{RM}=0,39)$ o multimorbilidad $(\beta=-1,797, \mathrm{RM}=0,17)$ reduce la probabilidad de declarar una salud buena. Esta situación se ha explorado entre los adultos mayores en México, ya que Gallegos et al. (2006) asociaron un ARS negativo entre las personas con EC.

Además, en el modelo 2 ajustado por covariables totales se estimó que, entre las mujeres, presentar limitación en una $\operatorname{ABVD}(\beta=-0,692, \mathrm{RM}=0,50)$ o dos o más ABVD $(\beta=-1,394, R M=0,25)$ disminuye la probabilidad de reportar una salud buena. En este sentido, las mujeres con síntomas depresivos mostraron menor probabilidad de presentar ARS bueno $(\beta=-1,466, R M=0,24)$. Los coeficientes para las variables de la situación laboral, el consumo de tabaco y la edad no fueron significativos $(p \geq 0,50)$.

La Tabla 3 muestra los resultados de las regresiones desarrolladas para los hombres. En cuanto al modelo 1 ajustado por covariables proporcionales se encontró que aquellos con una familia extendida tenían una mayor probabilidad de declarar un ARS bueno $(\beta=0,187, R M=1,21)$, un efecto similar al estimado en el modelo 2 ajustado por covariables totales, en el que pertenecer a una familia extendida incrementa la probabilidad de tener un ARS positivo $(\beta=0,196, \mathrm{RM}=1,22)$. 
TABLA 3

Estadísticos de ajuste y parámetros de los modelos logit para estimar los efectos de las covariables en los estados latentes definidos del ARS según la pregunta global. ENASEM 2012, 2015 y 2018

\begin{tabular}{|c|c|c|c|c|c|c|c|c|c|c|c|c|}
\hline \multirow{3}{*}{ Variables } & \multicolumn{6}{|c|}{$\begin{array}{l}\text { Modelo } 1 \text { ajustado por covariables } \\
\text { proporcionales }\end{array}$} & \multicolumn{6}{|c|}{ Modelo 2 ajustado por covariables totales } \\
\hline & \multicolumn{3}{|c|}{ Mujeres } & \multicolumn{3}{|c|}{ Hombres } & \multicolumn{3}{|c|}{ Mujeres } & \multicolumn{3}{|c|}{ Hombres } \\
\hline & $\beta$ & $\mathrm{EE}$ & RM & $\beta$ & $\mathrm{EE}$ & RM & $\beta$ & $\mathrm{EE}$ & RM & $\beta$ & $\mathrm{EE}$ & RM \\
\hline \multicolumn{13}{|l|}{$\begin{array}{l}\text { Situación } \\
\text { laboral (trabaja) }\end{array}$} \\
\hline No trabaja & - & - & - & - & - & - & $-0,222$ & 0,091 & 0,80 & $-0,060$ & 0,065 & 0,81 \\
\hline \multicolumn{13}{|l|}{$\begin{array}{l}\text { Situación } \\
\text { conyugal } \\
\text { (casado/unido) }\end{array}$} \\
\hline $\begin{array}{l}\text { Divorciado/ } \\
\text { viudo(a) }\end{array}$ & 0,101 & 0,077 & 1,11 & 0,111 & 0,105 & 1,12 & 0,166 & 0,077 & 1,18 & 0,250 & 0,085 & 1,25 \\
\hline Soltero(a) & 0,544 & 0,173 & 1,72 & 0,222 & 0,270 & 1,25 & 0,550 & 0,165 & 1,73 & 0,342 & 0,229 & 1,28 \\
\hline \multicolumn{13}{|c|}{$\begin{array}{l}\text { Arreglo } \\
\text { residencial } \\
\text { (familia nuclear) }\end{array}$} \\
\hline $\begin{array}{l}\text { Familia } \\
\text { extendida }\end{array}$ & 0,304 & 0,073 & 1,36 & 0,187 & 0,080 & 1,21 & 0,302 & 0,072 & 1,35 & 0,196 & 0,077 & 1,22 \\
\hline Vive solo(a) & 0,264 & 0,135 & 1,30 & 0,028 & 0,186 & 1,03 & 0,259 & 0,127 & 1,30 & 0,077 & 0,179 & 1,08 \\
\hline \multicolumn{13}{|c|}{$\begin{array}{l}\text { Multimorbilidad } \\
(\sin E C)\end{array}$} \\
\hline Una EC & $-1,077$ & 0,102 & 0,34 & - & - & - & $-0,955$ & 0,089 & 0,39 & $-0,883$ & 0,094 & 0,41 \\
\hline $2+E C$ & $-2,089$ & 0,123 & 0,12 & - & - & - & $-1,797$ & 0,109 & 0,17 & $-1,502$ & 0,116 & 0,22 \\
\hline \multicolumn{13}{|l|}{$\begin{array}{l}\text { Dependencia } \\
\text { funcional (sin } \\
\text { ABVD) }\end{array}$} \\
\hline Una ABVD & - & - & - & $-1,410$ & 0,143 & 0,24 & $-0,692$ & 0,108 & 0,50 & $-1,033$ & 0,136 & 0,36 \\
\hline $2+A B V D$ & - & - & - & $-1,620$ & 0,158 & 0,20 & $-1,394$ & 0,118 & 0,25 & $-0,963$ & 0,149 & 0,38 \\
\hline \multicolumn{13}{|l|}{$\begin{array}{l}\text { Síntomas } \\
\text { depresivos (no) }\end{array}$} \\
\hline Sí & - & - & - & - & - & - & $-1,446$ & 0,088 & 0,24 & $-1,432$ & 0,096 & 0,24 \\
\hline \multicolumn{13}{|l|}{$\begin{array}{l}\text { Consumo de } \\
\text { tabaco (nunca } \\
\text { ha fumado) }\end{array}$} \\
\hline Antes fumaba & 0,220 & 0,172 & 1,25 & 0,448 & 0,117 & 1,57 & 0,262 & 0,093 & 1,22 & 0,240 & 0,113 & 1,27 \\
\hline Fuma & 0,029 & 0,096 & 1,03 & $-0,133$ & 0,079 & 1,08 & 0,034 & 0,015 & 0,74 & $-0,045$ & 0,077 & 0,96 \\
\hline Edad & - & - & - & - & - & - & $-0,021$ & 0,015 & 0,99 & $-0,052$ & 0,017 & 0,95 \\
\hline Edad2 & - & - & - & - & - & - & 0,089 & 0,050 & 1,07 & 0,148 & 0,056 & 1,16 \\
\hline Punto de corte 1 & 3,713 & & & 3,179 & & & 4,708 & & & 4,698 & & \\
\hline $\begin{array}{l}\text { Punto de } \\
\text { corte } 2\end{array}$ & $-0,073$ & & & $-0,847$ & & & 0,020 & & & 0,315 & & \\
\hline Score-test (chi2) & 6,93 & & & 8,93 & & & & & & & & \\
\hline p>chi2 & 0,140 & & & 0,063 & & & & & & & & \\
\hline $\mathrm{BIC}$ & & 102,28 & & 13. & 586,52 & & & $.552,19$ & & & 462,47 & \\
\hline Observaciones & 3.298 & & & 2.613 & & & 3.298 & & & 2.613 & & \\
\hline
\end{tabular}

Fuente: Elaboración propia a partir de las 5.911 observaciones de entrevistados en el ENASEM 2012 y sobrevivientes a las rondas 2015 y 2018 (ENASEM, 2020).

Nota: $\beta=$ Coeficiente determinado por la función logit; $\mathrm{EE}=$ Error estándar, $\mathrm{RM}=$ razón de momios.

$\mathrm{BIC}=$ Bayesian Information Criterion.

En cuanto a las covariables de la salud de los hombres, tener dos y más EC $(\beta=-1,620$, $\mathrm{RM}=0,20$ ) se asocia con una menor probabilidad de un ARS bueno según el modelo 1 
ajustado por covariables proporcionales, estimaciones consistentes en el modelo 2 ajustado por covariables totales $(\beta=-1,502, \mathrm{RM}=0,22)$. Luego, las limitaciones para el desarrollo de las ABVD disminuyen la propensión a declarar un ARS positivo: modelo 1 ajustado por covariables proporcionales (una ABVD $\beta=-1,410, \mathrm{RM}=0,24$ ) y modelo 2 ajustado por covariables totales (una ABVD $\beta=-1,033, \mathrm{RM}=0,36$; dos o más $\mathrm{ABVD} \beta=-0,963$, $\mathrm{RM}=0,38)$. Además, en el modelo 1 ajustado por covariables proporcionales se estimó que ser exfumador incrementa la probabilidad de tener un ARS bueno $(\beta=0,448, R M=1,57)$.

Por otro lado, y aunque esto solo en el modelo 2 ajustado por covariables totales fue significativo, entre los hombres la presencia de síntomas depresivos se relaciona con una disminución en la probabilidad de declarar una salud buena $(\beta=-1,432, \mathrm{RM}=0,24)$. Además, se encontró que el aumento de la edad disminuye la probabilidad de un ARS bueno $(\beta=-0,052, R M=0,95)$. Los coeficientes de las variables de la situación laboral o de la situación conyugal no fueron significativos $(p \geq 0,50)$.

\section{Conclusiones}

El proceso de envejecimiento poblacional en México presenta fuertes retos en materia social, económica y en salud (WONG; MICHAELS-OBREGON; PALLONI, 2017). Entonces, el ARS se presenta como indicador para conocer los cambios e interacciones asociados con la salud de las personas mayores. Esta investigación analizó la dinámica del ARS en una muestra de personas mayores residentes en México como una forma de detectar trayectorias y variables que influyen en el ARS en forma longitudinal. En este estudio se construyeron MLM considerando que las personas evalúan los eventos que influyen en el ARS pasados y en prospectiva, es decir que se enfocó en la hipótesis de la trayectoria del ARS (IDLER; BENYAMINI, 1997).

Entre las mujeres y los hombres existiría un número igual de estados latentes, lo cual se traduce en que las trayectorias del ARS sean equivalentes (CAMELO et al., 2014; CHEN et al., 2016; VUORISALMI et al., 2006). Además, de entre los tres estados latentes con la variable ARS global, uno se podría relacionar con una salud regular, que además tenía las mayores probabilidades iniciales de transición y de permanencia entre estados.

En cuanto a los efectos estadísticos de las covariables en las trayectorias del ARS, se estimaron efectos significativos de los arreglos residenciales en el caso de las mujeres, en los que las que habitaban en una familia extendida tenían mayor probabilidad de tener un ARS bueno que aquellas que vivían en una familia nuclear o solas.

La multimorbilidad se asoció un efecto negativo sobre la probabilidad de un ARS positivo tanto en mujeres como en hombres. Al respecto, Rohlfsen y Jacobs Kronenfeld (2014) estimaron que las diferencias en las trayectorias del ARS se asocian con cambios en la salud motivados por la presencia de EC y, aunque de forma transversal, entre los adultos en México se ha encontrado evidencia de la asociación de las EC con ARS malos o muy malos (GALLEGOS et al., 2006; GONZÁLEZ-PICHARDO et al., 2014, PALLONI et al., 2003). La presencia de restricciones 
en el desarrollo de ABVD se asoció en forma negativa con un ARS positivo (JOHNSON; WOLINSKY, 1993; YOSHIMITSU et al., 2017). Además, los síntomas depresivos podrían tener una asociación negativa con mejores ARS. En particular, en el análisis de trayectorias del ARS quienes declaraban estados de salud positivos al inicio solían disminuir su valoración según la presencia de depresión (AMBRESIN et al., 2014). Por último, se asoció un efecto significativo de la edad al disminuir la probabilidad de un ARS bueno entre los hombres, lo cual ha sido indicado en otras investigaciones (PALLONI et al., 2003).

Los resultados de esta investigación buscan mostrar la importancia del ARS en torno a la salud de las personas de sesenta y más años de edad entrevistados en el ENASEM de 2012 y sobrevivientes a las rondas de 2015 y 2018. En este sentido, al comparar entre las defunciones y sobrevivientes capturadas entre rondas, se detectó un mayor porcentaje significativo de ARS malo entre quienes habían fallecido, mientras que la literatura señala que las personas con ARS malos tienen mayor riesgo de muerte (FALCONER; QUESNEL-VALLEÉ, 2016; SARGENT-COX et al., 2010; VUORISALMI et al., 2006). Este resultado permite preguntar sobre el ARS como predictor de la mortalidad entre los adultos mayores en México, lo cual podría determinarse con, por ejemplo, semiparametrizaciones de Cox o modelos estadísticos más complejos que el análisis descriptivo usado aquí (MILLER; WOLISNKY, 2007; SARGENT-COX et al., 2010; VUORISALMI et al., 2006).

Esta investigación presenta limitaciones que se relacionan con la selección de casos con información completa en las variables de interés, pues es posible que las personas sobrevivientes a las rondas del ENASEM tuvieran mejores condiciones cognitivas que quienes fallecieron entre ellas. Además, es importante apuntar que la atrición puede tener un efecto sobre las estimaciones longitudinales derivadas del análisis del ENASEM (OROZCO-ROCHA; WONG; MICHAELS OBREGÓN, 2018).

Entonces, quedan preguntas como si el deterioro cognitivo tiene efecto en el ARS de forma longitudinal. Además, el modelo estadístico elegido presenta alguna limitación relacionada con la existencia de un número limitado de estados latentes, así como la posibilidad de endogeneidad en los modelos logit proporcionales estimados.

A pesar de las limitaciones que este trabajo pueda tener, tiene fortalezas como estratificar los análisis por sexo (PALLONI et al., 2003; AGUILAR-PALACIO et al., 2017). También, analizar de forma longitudinal los cambios en el ARS entre personas adultas mayores puede servir como base para futuros estudios que analicen las propiedades del ARS como indicador de los cambios en la salud.

En conclusión, el ARS entre las mujeres y los hombres de edades avanzadas en México presenta trayectorias similares al evaluarse de forma global. Al no estimar efectos de las covariables en los estados latentes definidos, es necesario profundizar el análisis de los cambios y factores asociados con el ARS, así como también estudiar los efectos de variables como el aislamiento social (PINILLOS-FRANCO; KAWACHI, 2017). 


\section{Referencias}

AGUILAR-NAVARRO, S G.; FUENTES-CANTÚ, A.; ÁVILA-FUNES, J. A.; GARCÍA-MAYO, E. J. Validez y confiabilidad del cuestionario del ENASEM para la depresión en adultos mayores. Salud Pública de México, v. 49, p. 256-262, 2017.

AGUILAR-PALACIO, I.; CARRERA-LASFUENTES, P.; SÁNCHEZ-RECIO, R.; ALONSO, J. P.; RABANAQUE, M. J. Recession, employment and self-rated health: a study on the gender gap. Public Health, n. 154, p. 44-50, 2017.

AMBRESIN, G.; CHONDROS, P.; DOWRICK, C.; HERRMAN, H.; GUNN, J. M. Self-rated health and long-term prognosis of depression. Annals of Family Medicine, v. 12, n. 1, p. 57-65, 2014.

AROKIASAMY, P.; UTTAMACHARYA, K. J. Multi-morbidity functional limitations, and self-rated health among older adults in India: cross-sectional analysis of LASI Pilot Survey, 2010. SAGE Open, v. 5, n. 1, 2015.

BARTOLUCCI, F.; FARCOMENI, A.; PENNONI, F. Latent Markov models for longitudinal data. Boca Raton: Chapman y Hall/CRC, 2012.

BARTOLUCCI, F.; PANDOLFI, S.; PENNONI, F. LMest: An R Package for latent Markov models for longitudinal categorical data. Journal of Statistical Software, v. 81, n. 4, p. 1-38, 2017.

BERDAHL, T. A.; MCQUILLAN, J. Self-rated health trajectories among married Americans: do disparities persist over 20 years? Journal of Aging Research, article ID 1208598, 2018.

CAMELO, L. V.; GIATTI, L.; BARRETO, S. M. Subjective social status, self-rated health and tobacco smoking. Brazilian Longitudinal Study of Adult Health (ELSA-Brasil). Journal of Health Psychology, v. 91, n. 11, p. 1388-1399, 2014.

CEPAL - Comisión Económica para América Latina y el Caribe; OIT - Organización Internacional del Trabajo. Coyuntura laboral en América Latina y el Caribe. La inserción laboral de las personas mayores: necesidades y opciones. Santiago, Chile: Cepal/OIT, 2018.

CHEN, S.; WHITSON, H.; QUIÑNES, A.; THIELKE, S. Comparative health and self-rated health are equivalently associated with health indicators among older adults. Journal of Clinical Epidemiology, v. 70, p. 279-280, 2016.

CONAPO - Consejo Nacional de Población. Proyecciones de población de México y de las entidades federativas 2016-2050. México: Conapo, 2018. Disponible en: https://datos. gob. $\mathrm{mx} /$ busca/dataset/proyecciones-de-la-poblacion-de-mexico-y-de-las-entidadesfederativas-2016-2050.

ENASEM - Estudio Nacional de Salud y Envejecimiento en México. Archivos de Datos y Documentación (uso público). Estudio Nacional de Salud y Envejecimiento en México (Datos Brutos), 2020. Disponible en: www.ENASEM.org. Acceso en: 10 ago. 2020.

FALCONER, J.; QUESNEL-VALLEE, A. Pathway from poor self-rated health to mortality: explanatory power of disease diagnosis. Social Science \& Medicine, n. 190, p. 227-236, 2016.

FRANCO-MARINA, F. Adult smoking trends in Mexico: an analysis of the Mexican National Addiction Surveys. Salud Pública de México, v. 49, supl. 2, p. S137-S146, 2007.

GALLEGOS, K.; GARCÍA, C.; DURAN, C.; REYES, H.; DURAN, L. Self-perceived health status: an approach of the elderly in Mexico. Revista de Saúde Pública, v. 40, n. 5, p. 792-801, 2006.

IDLER, E. L.; BENYAMINI, Y. Self-rated health and mortality: a review of twenty-seven community studies. Journal of Health and Social Behavior, v. 38, n. 1, p. 21-37, 1997. 
INEGI - Instituto Nacional de Estadística y Geografía. Encuesta Intercensal 2015. Tabulados básicos. Disponible en: https://www.inegi.org.mx/programas/intercensal/2015/default. html\#Tabulados.

JOHNSON, R. J.; WOLINSKY, F. D. The structure of health status among older adults: disease, disability, functional limitation, and perceived health. Journal of Health and Social Behavior, v. 34, n. 2, p. 105-121, 1993.

JOUNG, I. M.; STRONKS, K.; VAN DE MHEEN, H.; MACKENBACH, J. P. Health behaviors explain part of the differences in self-reported health associated with partner/marital status in the Netherlands. Journal of Epidemiology and Community Health, v. 49, n. 5, p. 482-488, 1995.

JYLHÄ, M. Self-rated health and subjective survival probabilities as predictors of mortality. In: ROGERS, R. G.; CRIMMINS, E. M. (ed.). International handbook of adult mortality. The Netherlands: Springer, 2011.

KAPLAN, G. A.; CAMACHO, T. Perceived health and mortality: a nine-year follow-up of the human population laboratory cohort. American Journal of Epidemiology, v. 117, n. 3, p. 292-306, 1983.

KATZ, S.; FORD, A.; MOSKOWITZ, R.; JACKSON, B.; JAFFE, M. Studies of illness and the aged: the index of ADL, a standardized measure of biological and psychological function. JAMA, n. 185, p. 914-919, 1963.

LEE, S.; SCHWARZ, N.; GOLDSTEIN, L. S. Culture-sensitive question order effects of self-rated health between older Hispanic and non-Hispanic adults in the United States. Journal of Aging Health, v. 26, n. 5, p. 860-883, 2014.

LI, M.; HARRIS, I.; LU, K. Differences in proxy-reported and patient-reported outcomes: assessing health and functional status among medicare beneficiaries. BMC Medical Research Methodology, n. 15, p. 62-72, 2015.

MANRIQUE-ESPINOZA, B.; SALINAS-RODRIGUEZ, A.; MORENO-TAMAYO, M.; ACOSTA-CASTILLO, I.; SOSA-ORTIZ, A.; GUTIERREZ-ROBLEDO, L. M.; TELLEZ-ROJO, M. Condiciones de salud y estado funcional de los adultos mayores en México. Salud Pública de México, v. 55, supl. 2, p. S323-S331, 2013.

MARTELL-CLAROS, N.; ARANDA, P.; GONZÁLEZ-ALBARRÁN, O.; DALFÓ-BAQUÉ, A.; DOMÍNGUEZSARDIÑANA, M.; DE LA CRUZ, J. A. C. Perception of health and understanding of cardiovascular risk among patients with recently diagnosed diabetes and/or metabolic syndrome. European Journal of Preventive Cardiology, v. 20, n. 1, p. 21-28, 2011.

MEADOWS, S. O. Family structure and fathers' well-being: trajectories of self-rated and mental health. Journal of Health and Social Behavior, v. 50, n. 2, p. 115-131, 2009.

MIILUNPALO, S.; VUORI, I.; OJA, P.; PASANEN, M.; URPONEN, H. Self-rated health status as a health measure: the predictive value of self-reported health status on the use of physician services and on mortality in the working-age population. Journal of Clinical Epidemiology, v. 50, n. 5, p. 517-528, 1997.

MILLER, T. R.; WOLISNKY, F. D. Self-rated health trajectories and mortality among older adults. The Journals of Gerontology Series B: Psychological and Social Sciences, v. 62, n. 1, p. S22-S27, 2007.

MOLINA, T. Adjusting for heterogeneous response thresholds in cross-country comparisons of self-reported health. The Journal of the Economics of Ageing, n. 10, p. 1-20, 2017.

OCAMPO, M. Self-rated health: importance of use in elderly adults. Colombia Médica, n. 41, p. 275-289, 2010. 
OROZCO-ROCHA, K.; WONG, R.; MICHAELS OBREGÓN, A. Atrición en encuestas de panel en México: la Encuesta Nacional sobre Salud y Envejecimiento en México. Datos y Espacio. Revista Internacional de Estadística y Geografía, v. 9, n. 1, p. 54-71, 2018.

OVERLAND, S.; GLOZIER, N.; MAELAND, J. G.; AARO, L. E.; MIKLETUN, A. Employment status and perceived health in the Hordaland Health Study (HUSK). BMC Public Health, n. 6, article 219, 2006.

PALLONI, A.; SOLDO, B.; WONG, R.; MCENIRY, M. Health status in a national sample of elderly Mexicans. Madison, WI: Center for Demography and Ecology, University of Wisconsin-Madison, 2003. (CDE Working Paper, n. 2003-03). Disponible en: https://www.researchgate.net/ publication/242118372_Health_Status_in_a_National_Sample_of_Elderly_Mexicans.

PICAZZO-PALENCIA, E. Depression and mobility among older adults in Mexico: ENSANUT 2012. Hispanic Health Care International, v. 14, n. 2, p. 94-98, 2016.

PINILLOS-FRANCO, S.; KAWACHI, I. The relationship between social capital and self-reported health: a gendered analysis of 17 European countries. Social Science \& Medicine, n. 219, p. 30-35, 2017.

ROHLFSEN, L. S.; JACOBS KRONENFELD, J. Trajectories of self-rated health in middle and old age: an examination of differential exposure and differential vulnerability. Journal of Aging and Health, v. 26, n. 4, p. 637-662, 2014.

SALINAS, J. Preventive health screening utilization in older Mexicans before and after healthcare reform. Salud Pública de México, v. 57, (sup. I), p. S70-S78, 2015.

SARGENT-COX, K. A.; ANSTEY, K. J.; LUSZCZ, M. A. The choice of self-rated health measures matter when predicting mortality: evidence from 10 years follow-up of the Australian longitudinal study of ageing. BMC Geriatrics, n. 10, p. 18, 2010.

SUH, J. K.; DOCTOR, J. Self-rated health measures with different reference points and mortality. Value in Health, v. 16, n. 3, p. A265, 2013.

VUORISALMI, M.; LINTONEN, T.; JYLHÄ, M. Global self-rated health data from a longitudinal study predicted mortality better than comparative self-rated health in old age. Journal of Clinical Epidemiology, v. 18, n. 3, p. 211-217, 2006.

WEXLER, D. J.; PORNEALA, B.; CHANG, Y.; HUANG, E. S.; HUFFMAN, J. C.; GRANT, R. W. Diabetes differentially affects depression and self-rated health by age in the U.S. Diabetes Care, v. 35, n. 7, p. 1575-1577, 2012.

WOLINSKY, F. D.; TIERNEY, W. M. Self-rated health and adverse health outcomes: an exploration and refinement of the trajectory hypothesis. The Journals of Gerontology Series B: Psychological and Social Sciences, v. 53, n. 6, p. 336-S340, 1998.

WONG, R.; MICHAELS-OBREGON, A.; PALLONI, A. Cohort profile: the Mexican health and aging study. International Journal of Epidemiology, v. e2, p. 1-10, 2017.

WONG, R.; MICHAELS-OBREGON, A. Mexican health and aging study. In: GU, D.; DUPRE, M. E. (ed.). Encyclopedia of gerontology and population aging. Suiza: Springer International Publishing, 2019.

YOSHIMITSU, K.; TABIRA, T.; KUBOTA, M.; IKEDA, Y.; INOUE, K.; AKASAKI, Y. Factors affecting the self-rated health of elderly individuals living alone: a cross-sectional study. BMC Research Notes, n. 10, p. 512-518, 2017.

ZAJACOVA, A.; HUZURBAZAR, S.; TODD, M. Gender and the structure of self-rated health across the adult life span. Social Science \& Medicine, n. 187, p. 58-66, 2017. 


\title{
Sobre los autores
}

Daniel Lozano Keymolen es Doctor en Estudios de Población por El Colegio de México A. C. Profesor de tiempo completo en el Centro de Investigación y Estudios Avanzados de la Población de la Universidad Autónoma del Estado de México.

Sergio Cuauhtémoc Gaxiola Robles Linares es Doctor en Estudios de Población por El Colegio de México A. C. Profesor de tiempo completo en el Centro de Investigación y Estudios Avanzados de la Población de la Universidad Autónoma del Estado de México.

Alejandro Martínez Espinosa es Doctor en Estudios de Población por El Colegio de México A. C. Profesor de tiempo completo en el Centro de Investigación y Estudios Avanzados de la Población de la Universidad Autónoma del Estado de México.

\section{Dirección para correspondencia}

\author{
Daniel Lozano Keymolen \\ Paseo Tollocan s/n, Cerro de Coatepec, Ciudad Universitaria \\ 50110 - Toluca de Lerdo, Estado de México, México \\ Sergio Cuauhtémoc Gaxiola Robles Linares \\ Paseo Tollocan s/n, Cerro de Coatepec, Ciudad Universitaria \\ 50110 - Toluca de Lerdo, Estado de México, México \\ Alejandro Martínez Espinosa \\ Paseo Tollocan s/n, Cerro de Coatepec, Ciudad Universitaria \\ 50110 - Toluca de Lerdo, Estado de México, México
}

\section{Resumo}

Autorrelato de saúde em idosos no México, 2012-2018

O objetivo desta pesquisa é analisar os efeitos de certos fatores sociodemográficos e de saúde nas trajetórias de autorrelato de saúde de idosos no México. Com dados do Estudo Nacional de Saúde e Envelhecimento no México 2012-2018, as trajetórias foram estimadas usando modelos de estado latente de Markov. Os resultados indicam que existem três trajetórias, tanto para mulheres quanto para homens, que foram constantes mesmo ajustando para variáveis sociodemográficas e de saúde. No entanto, arranjo residencial, multimorbidade ou sintomas depressivos tiveram efeitos negativos sobre a probabilidade de um autorrelato positivo de saúde. Esses resultados sugerem que, embora a análise dos fatores associados às trajetórias definidas deva ser aprofundada, arranjos residenciais, múltiplas doenças crônicas ou depressão têm efeitos longitudinais na autoavaliação da saúde de idosos no México.

Palavras-chave: Autorrelato de saúde. Estados latentes. Envelhecimento. Doenças crônicas. México. 


\section{Abstract}

Self-assessed health of older adults in Mexico, 2012 - 2018

The objective of this research was to analyze the effects of certain sociodemographic and health factors on the self-assessed health trajectories of older adults in Mexico. With data from the Mexican Health and Aging Study 2012-2018, trajectories were estimated using Markov latent state models. The results indicate that, in both women and men, there are three trajectories which were constant, even adjusting for sociodemographic and health variables. However, residential arrangement, multimorbidity, or depressive symptoms had negative effects on the likelihood of a positive self-report of health. These results suggest that, although the factors associated with the defined trajectories of self-rated health, should be analyzed further, residential arrangements, multiple chronic illnesses or depression have longitudinal effects on the self-assessed health of older adults in Mexico.

Keywords: Self-rated health. Latent states. Aging. Chronic diseases. Mexico.

Recibido para publicación en 28/10/2020 Aceptado para publicación en 03/06/2021 